\title{
Time-course RNA-seq analysis reveals stage-specific and melatonin-triggered gene expression patterns during the hair follicle growth cycle in Capra hircus
}

\author{
Chun $\mathrm{Li}^{1 \dagger}$, Cong Feng ${ }^{2 \dagger}$, Guangyuan $\mathrm{Ma}^{2 \dagger}$, Shaoyin $\mathrm{Fu}^{3}$, Ming Chen ${ }^{2,4^{*}}$, Wenguang Zhang ${ }^{5^{*}}$ and Jinquan $\mathrm{Li}^{5^{*}}$
}

\begin{abstract}
Background: Cashmere goat is famous for its high-quality fibers. The growth of cashmere in secondary hair follicles exhibits a seasonal pattern arising from circannual changes in the natural photoperiod. Although several studies have compared and analyzed the differences in gene expression between different hair follicle growth stages, the selection of samples in these studies relies on research experience or morphological evidence. Distinguishing hair follicle growth cycle according to gene expression patterns may help to explore the regulation mechanisms related to cashmere growth and the effect of melatonin from a molecular level more accurately.
\end{abstract}

Results: In this study, we applied RNA-sequencing to the hair follicles of three normal and three melatonin-treated Inner Mongolian cashmere goats sampled every month during a whole hair follicle growth cycle. A total of 3559 and 988 genes were subjected as seasonal changing genes (SCGs) in the control and treated groups, respectively. The SCGs in the normal group were divided into three clusters, and their specific expression patterns help to group the hair follicle growth cycle into anagen, catagen and telogen stages. Some canonical pathways such as Wnt, TGF-beta and Hippo signaling pathways were detected as promoting the hair follicle growth, while Cell adhesion molecules (CAMs), Cytokine-cytokine receptor interaction, Jak-STAT, Fc epsilon RI, NOD-like receptor, Rap1, PI3K-Akt, cAMP, NFkappa B and many immune-related pathways were detected in the catagen and telogen stages. The PI3K-Akt signaling, ECM-receptor interaction and Focal adhesion were found in the transition stage between telogen to anagen, which may serve as candidate biomarkers for telogen-anagen regeneration. A total of 16 signaling pathways, 145 pathway mRNAs, and 93 IncRNAs were enrolled to construct the pathway-mRNA-IncRNA network, which indicated the function of IncRNAs through interacting with their co-expressed mRNAs. Pairwise comparisons between the control and melatonin-treated groups also indicated 941 monthly differentially expressed genes (monthly DEGs). These monthly DEGs were mainly distributed from April and September, which revealed a potential signal pathway map regulating the anagen stage triggered by melatonin. Enrichment analysis showed that Wnt, Hedgehog, ECM,

\footnotetext{
*Correspondence: mchen@zju.edu.cn; atcgnmbi@yahoo.com.cn; lijinquan_ nd@126.com

${ }^{\dagger}$ Chun Li, Cong Feng and Guangyuan Ma contributed equally to this

work.

${ }^{2}$ College of Life Sciences, Zhejiang University, Hangzhou 310058, China

${ }^{5}$ College of Animal Science, Inner Mongolia Agricultural University,

Hohhot 010018, China

Full list of author information is available at the end of the article
}

(C) The Author(s) 2022. Open Access This article is licensed under a Creative Commons Attribution 4.0 International License, which permits use, sharing, adaptation, distribution and reproduction in any medium or format, as long as you give appropriate credit to the original author(s) and the source, provide a link to the Creative Commons licence, and indicate if changes were made. The images or other third party material in this article are included in the article's Creative Commons licence, unless indicated otherwise in a credit line to the material. If material is not included in the article's Creative Commons licence and your intended use is not permitted by statutory regulation or exceeds the permitted use, you will need to obtain permission directly from the copyright holder. To view a copy of this licence, visit http://creativecommons.org/licenses/by/4.0/. The Creative Commons Public Domain Dedication waiver (http://creativeco mmons.org/publicdomain/zero/1.0/) applies to the data made available in this article, unless otherwise stated in a credit line to the data. 
Chemokines and NF-kappa B signaling pathways may be involved in the regulation of non-quiescence and secondary shedding under the influence of melatonin.

Conclusions: Our study decoded the key regulators of the whole hair follicle growth cycle, laying the foundation for the control of hair follicle growth and improvement of cashmere yield.

Keywords: Hair follicle growth cycle, Cashmere goat, RNA-seq, Melatonin, Signaling pathway

\section{Background}

The Inner Mongolian cashmere goat (Capra hircus), an excellent cashmere goat breed in China, is famous for producing cashmere with superior quality and high yield. The cashmere goat has two different fibrous hair structures: thick and coarse hairs forming the guard layer and soft hairs forming the ground cashmere. The cashmere comes from secondary hair follicles (SHFs) in the skin [1], and the coarse hair comes from primary hair follicles $[2,3]$. The cashmere obtained from goats is specially used for production of expansive textile products [4]. Under the periodic changes of the natural photoperiod, the growth of cashmere in the Inner Mongolian cashmere goat presents a seasonal pattern. Normally, a typical hair follicle growth cycle starts in July and stops in March of the following year with shedding of the cashmere at April [5].

Melatonin is an important mediator between photoperiod and the hair follicle growth. The cyclical fluctuation of melatonin levels directly affects the hair follicle growth [6]. Previous studies have shown that the use of exogenous melatonin could stimulate hair follicle growth. However, a number of studies have shown that the implantation and duration of melatonin may lead to earlier shedding of cashmere [6-9], or increase production by promoting the growth of cashmere $[10,11]$. Although the positive role on cashmere growth of exogenous melatonin has been confirmed, previous experiments have not been able to dynamically show the gene expression profiles related to the whole cycle of hair follicle growth and the potential effect of exogenous melatonin on the hair follicle growth cycle.

The hair follicle growth cycle of the cashmere goat can be divided into three phases: anagen, catagen and telogen, which are regulated by specific genetic regulators [5]. For Inner Mongolian cashmere goats, the anagen phase of SHFs is from April to November, the catagen phase is from December to January, and the telogen phase is from February to March [12]. SHFs continue to grow during the anagen phase and the cashmere continues to elongate. At the catagen phase, hair follicle cells undergo apoptosis and the growth rate of cashmere slows down to stop. Finally, SHFs enter the telogen phase and are accompanied by the shedding of cashmere [13]. With the rapid development of high-throughput sequencing technology, some regulatory factors and signaling pathways involved in the hair follicle cycle have been found through differential expression and functional enrichment analysis. These well-known regulatory molecules and signaling pathways include Wnt/ $\beta$ - catenin [14-16], bone morphogenetic proteins (BMPs) [17, 18], sonic hedgehog $(\mathrm{SHH})$ [19], notch [20], fibroblast growth factors (FGFs) [21], transforming growth factors (TGFs) [22] and keratin-associated proteins (KRTAPs) [5, 23], etc.

Long noncoding RNAs (lncRNAs) are a type of RNAs that are longer than 200 nucleotides but do not encode proteins. However, lncRNAs can regulate the expression of protein-coding genes at various levels to influence biological processes, including epigenetic regulation, transcriptional regulation and posttranscriptional regulation [24]. The regulation mechanisms of lncRNAs in hair growth have been reported by some recent studies. For example, several important hair follicle development signals (lncRNAs and mRNAs) are involved in primary wool follicle induction in carpet sheep [25]. Yin et al. [26] indicated that lncRNA-599,554 contributes the inductive property of dermal papilla cells in cashmere goat, which might be achieved through sponging chi-miR-15b-5p to promote the WNT3A expression. Wang et al. [27, 28] integrated analysis of lncRNA, miRNA and mRNA in cashmere goat skin during anagen and telogen stages and revealed potential ceRNA regulatory networks. Sulayman et al. [29] performed a comprehensive analysis of lncRNA and mRNA expression profiles during sheep fetal and postnatal hair follicle development and demonstrated that the interaction between lncRNA and their target genes may regulate the development of hair follicles. However, the roles of IncRNAs in controlling the whole hair follicle growth cycle have not been well described.

To clarify the regulatory mechanism of the hair follicle growth cycle and gain insight into the gene regulatory network perturbed by exogenous melatonin, we use RNA-seq analysis to investigate the expression patterns of seasonal changing genes (SCGs) among different gene clusters. The interactions between lncRNAs and mRNAs were also explored using co-expression network analysis. The monthly differentially expressed genes obtained from pairwise comparisons between the control and melatonin-treated groups were detected to identify the key regulators associated with the growth of secondary hair 
follicles and reveal potential signaling pathways which may be involved in melatonin-affected growth patterns.

\section{Results}

\section{Transcriptome analysis and differential gene expression} overview

A total of 72 samples spanning 12 months were analyzed in the control (D) and melatonin (M) groups (Fig. 1A). The experiments produced 2,959,842,480 clean reads in total ( 926G). A total of 22,404 genes were detected from reads counting through the RNA-seq data analysis (Additional file 1). A total of 2365 novel lncRNA genes were identified after the protein-coding-potential test (Additional file 2). Using DESeq2 time-series data analysis, 3559 and 988 genes were subjected as SCGs by fold change $\geq 2$ and adjusted $p$-value $\leq 0.05$ in the $\mathrm{D}$ and $\mathrm{M}$ groups, respectively (Fig. 1B) (Additional file 3 and Additional file 4). Among these SCGs, a total of 345 novel

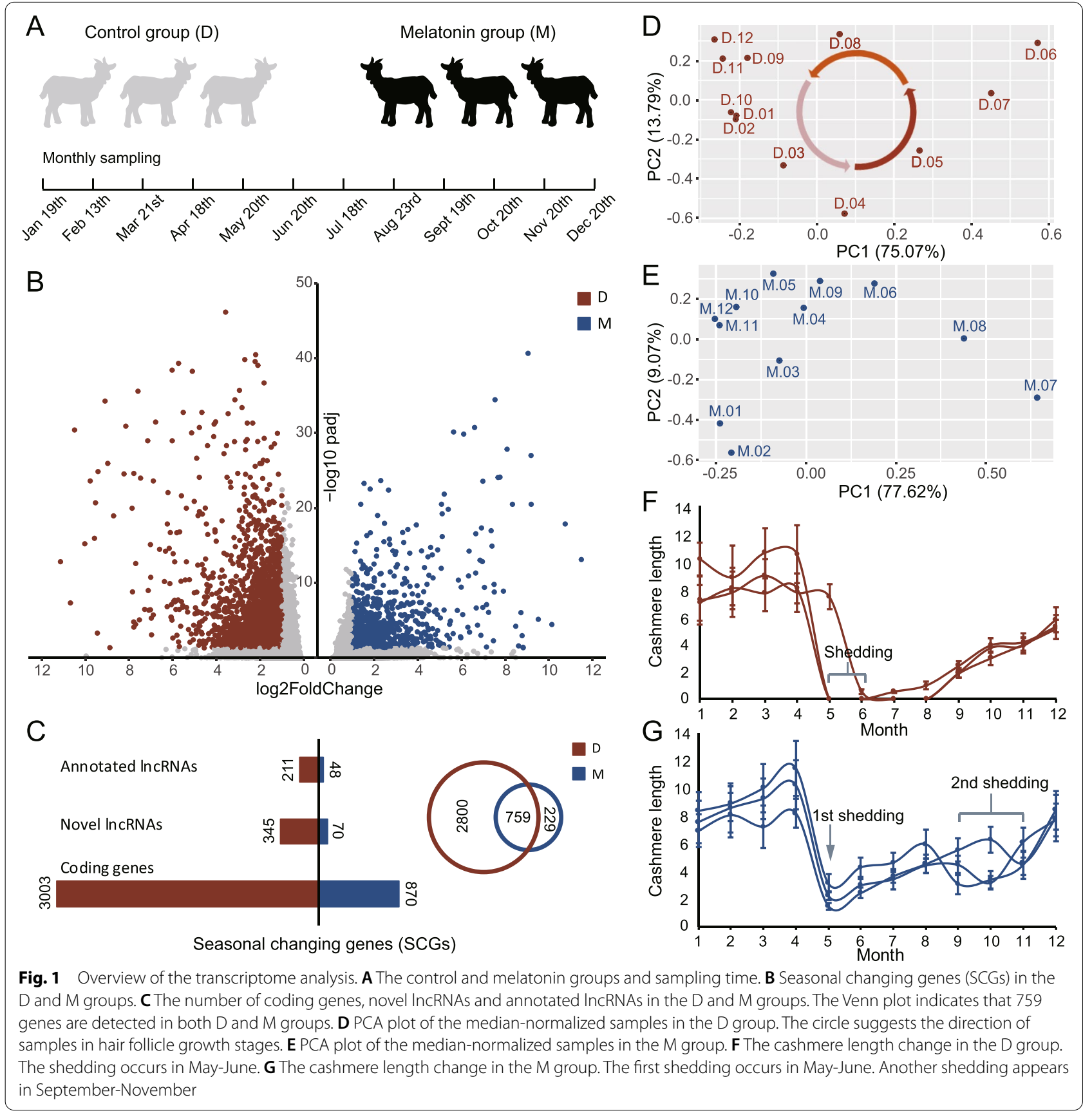


lncRNAs and 211 annotated lncRNAs were detected in the D group. We found that SCGs in the M group were much less than those in the D group, and 76.8\% (759) of the SCGs in the M group also existed in the $\mathrm{D}$ group (Fig. 1 C). According to the PCA diagrams in Fig. 1D and $\mathrm{E}$, the samples of the $\mathrm{D}$ group showed a certain periodic distribution, but the sample distribution was disturbed after the melatonin treatment. It can be seen in Fig. $1 \mathrm{~F}$ and $\mathrm{G}$ that the goats in two groups shed the cashmere in May, while goats in the M group had another shedding in September-November. An interesting finding is that in the $\mathrm{M}$ group, new cashmere has grown during the shedding in May, that is, the SHFs may not enter the telogen phase and the hair follicle growth cycle has restarted in advance (Fig. 1G).

\section{Stage-specific gene expression dynamics in the hair follicle growth cycle}

The correct division of the hair follicle growth cycle is the basis for subsequent identification and analysis of regulatory factors. The existing studies are mostly based on morphological evidence or prior knowledge. A recent study divided the hair follicle growth cycle into anagen, catagen and telogen phases based on skin tissue sections and transcriptome data [5]. However, their analysis is based on static differential expression analysis, the dynamic change of gene expression has not been well studied. In our study, we analyzed the time-series transcriptome data covering the entire hair follicle growth cycle and obtained 3559 SCGs. Through WGCNA analysis, three key gene clusters (DC1, DC2 and DC3) that may be involved in the growth cycle of villi were identified in the D group (Fig. 2 A). The detailed genes of three clusters are listed in Additional file 5, which encompass $48.2 \%$ (1717) of the SCGs detected in the D group, including 91 annotated lncRNAs and 131 novel lncRNAs (Fig. 2B). Combining the expression patterns and functional enrichment results of three clusters (Fig. $2 \mathrm{~A}$ and $\mathrm{C}$ ), we inferred the effects of these clustered genes on the hair follicle growth cycle. The genes in DC1 may promote cashmere growth and the genes in DC2 may be related to the regression of the SHFs. There may be an antagonistic relationship between $\mathrm{DC} 1$ and $\mathrm{DC} 2$, which can also be inferred from the gene expression trends in Fig. 2D. The gene expression in DC1 began to be up-regulated in April and reached a peak in October and started to be downregulated, while the gene expression pattern in $\mathrm{DC} 2$ was exactly the opposite. In addition, it can also be found that the high expression of DC3 occurs when the expression of $\mathrm{DC} 1$ genes begins to be up-regulated and the expression of DC2 genes begins to down-regulate. Based on the above analysis, we divided the hair follicle growth cycle into three stages associated with three gene clusters: (1) anagen (April-October); (2) catagen and telogen (October-December and January-April); (3) telogen-anagen regeneration (February-May). The detailed functional analysis of these three clusters and their relationships with the hair follicle growth cycle are described below.

\section{The DC1 cluster shows high expression in anagen progression stage}

A total of 394 genes in DC1 (including 22 annotated lncRNAs and 52 novel lncRNAs) are positively correlated with the anagen progression stage (April-October), which may be involved in promoting the cashmere growth (Fig. $2 \mathrm{~A}$ and D). Some canonical pathways such as the Wnt, TGF-beta and Hippo signaling pathways, which have been proven to be closely related to hair growth $[16$, 30, 31] are also enriched in DC1 (Fig. 2 C) (Additional file 6). Three genes of the Wnt family WNT2/WNT2B/ WNT11, together with their receptors FZD3 and FZD10 are involved in the regulation of the SHF development in cashmere goats. Lymphoid enhancer binding factor-1 (LEF1) is an essential transcription factor in the Wnt signaling, and it was strongly expressed during the anagen progression stage in this study. Its function in hair cell differentiation and follicle morphogenesis has already been discussed [32]. Genes in the TGF-beta signaling (BMP2, BMP8A, BAMBI and SMAD6) also showed similar patterns with the Wnt family. It is found that the genes enriched in the Hippo signaling overlap with those in the Wnt and TGF-beta signaling, indicating the crosstalk between Wnt and TGF-beta signaling formed by the joint regulation of downstream pathways [16, 33]. In addition, some downstream regulatory mechanisms of hair growth and cycling were also confirmed in this study. For example, the homeobox transcription factor DLX3 and one of its regulating genes, HOXC13, were also highly expressed in the anagen stage. The regulatory cascade positions DLX3 downstream of Wnt signaling and regulates other transcription factors related to hair follicle (HF) differentiation (such as HOXC13) [34]. KRTs (KRT26, KRT35, KRT36, KRT39, KRT6A, KRT74 and KRT84) and KRTAPs (KRTAP3-1 and KRTAP11-1) in DC1 were also associated with HF development [35]. $\mathrm{SHH}$ and its receptor PTCH2 in the sonic hedgehog (Shh) signaling pathway were also found in DC1. The function of Shh signaling is indicated as an essential regulator for controlling ingrowth and morphogenesis of the HFs [36-38], but it is not necessary for initiating the HF development [36].

\section{The DC2 cluster prefers to be highly expressed in catagen and telogen}

A total of 919 genes in DC2 (including 45 annotated lncRNAs and 53 novel lncRNAs) were up-regulated from 

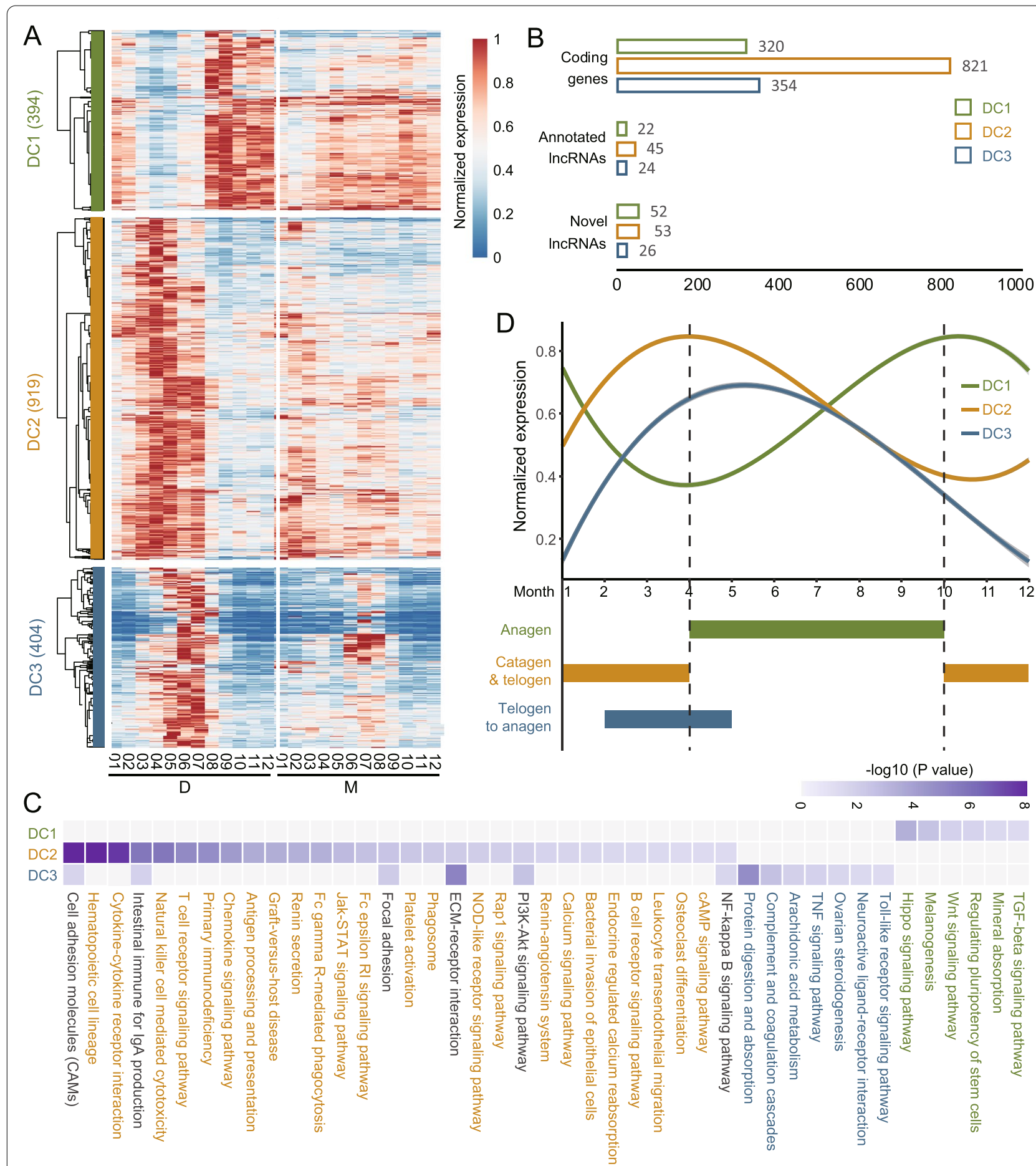

Fig. 2 Stage-specific gene expression patterns and pathway enrichment. A Heatmap of the expression level of three D clusters (DC1, DC2 and DC3) in D and M groups. B The number of coding genes, annotated IncRNAs and novel IncRNAs in three D clusters. C KEGG pathway enrichment of the genes in DC1, DC2 and DC3. The pathways shared by DC2 and DC3 are shown in black. D The expression trends of three gene clusters. The hair follicle growth cycle is divided into three stages according to the expression patterns 
October to April of the following year (Fig. $2 \mathrm{~A}$ and 2D), which corresponded to the catagen and telogen phases of the hair follicle growth cycle. It should be pointed out that due to the limitation of sampling interval, the catagen and telogen stages were not further distinguished in this study. The expression pattern of DC2 is negatively correlated with $\mathrm{DC} 1$, suggesting that there may be an antagonistic relationship between the genes in DC2 and DC1. DC2 genes are mainly enriched in pathways such as Cell adhesion molecules (CAMs), Cytokine-cytokine receptor interaction, Jak-STAT, Fc epsilon RI, NOD-like receptor, Rap1, PI3K-Akt, cAMP, NF-kappa B and many immune-related pathways (Fig. 2 C) (Additional file 6). A previous study also found that differentially expressed genes between anagen and telogen SHF-derived dermal papilla cells of the cashmere goats were also enriched in CAMs and Cytokine-cytokine receptor interaction pathways [1]. A number of interleukin (IL) superfamily genes were involved in the enriched pathways of DC2, such as the IL1 family (IL7 and IL18), IL10, IL15, and IL receptors (IL2RG, IL3RA, IL6R, IL7R, IL11RA and IL20RA). The CC chemokine subfamily (CCL5, CCL21, CCL22 and CCL26) and receptors (CCR4 and CCR6) were also found in the DC2 cluster. These chemokines are mainly involved in cell migration, immunity and inflammation [39]. During the HF regression, these chemokines may guide the migration of immune cells such as dendritic cells [40] and Regulatory T cells [41], thereby regulating the immune response to apoptotic cells [42]. The JAK3 and STAT4 genes in the JAK-STAT signaling were highly expressed in catagen and telogen, which have been found to maintain HF stem cell quiescence and inhibit hair growth [43-45]. In addition, Dickkopf1 (DKK1), a Wnt signaling inhibitor, was also found in DC2. DKK1 has been strongly suggested to promote regression of HFs by suppressing Wnt/ $\beta$-catenin signaling and inducing apoptosis in follicular keratinocytes $[46,47]$.

\section{The DC3 cluster is specifically expressed in telogen-anagen regeneration stage}

DC3 cluster contains a total of 404 genes (including 24 annotated lncRNAs and 26 novel lncRNAs) and shows specific high expression during the transition period from February to May (Fig. $2 \mathrm{~A}$ and D). Due to the overlap of the early anagen and telogen-anagen regeneration stages, the genes of DC3 and DC2 were partially enriched in several same pathways like CAMs, Focal adhesion, extracellular matrix (ECM)-receptor interaction, PI3KAkt and NF-kappa B signaling (Fig. 2 C) (Additional file 6). The PI3K-Akt signaling has been proved to be essential for HF regeneration [48, 49]. A previous study found that the Toll-Like Receptor 3 (TLR3) activated by a dsRNA was able to promote HF regeneration [50].
Several collagen genes (COL1A1, COL1A2, COL3A1, COL5A2, COL6A3, COL6A5 and COL6A6) in the PI3KAkt signaling, ECM-receptor interaction and Focal adhesion were found in DC3. These collagen genes may serve as candidate biomarkers for telogen-anagen regeneration. For example, a kind of self-assembling peptide hydrogel scaffold was used to build the ECM environment in vitro to promote HF regeneration [51].

\section{Pathway crosstalk through mRNA-IncRNA co-expression network in hair follicle growth cycle}

A total of 16 signaling pathways, 145 pathway genes, and 93 co-expressed lncRNAs (Pearson correlation $\geq 0.8$ ) (Additional file 7) are enrolled in this pathway-mRNAlncRNA network (Fig. 3). It can be seen that the DC1 subnet has no positive correlation with $\mathrm{DC} 2$ and $\mathrm{DC} 3$, which can be explained by the distinct expression patterns in Fig. 2D and indicates possible antagonistic relationship between DC1 and DC2. The telogen-anagen transition phase of DC3 overlaps with the telogen stage, so the DC3 subnet is closely connected to DC2. Through this network, the function of lncRNAs can be inferred by their co-expressed coding genes. Three novel lncRNAs (LNC.6206, LNC.8064 and LNC.16,941) were highly connected (by more than 6 coding genes) in the DC1 subnet, indicating that they may mediate in promoting $\mathrm{HF}$ development. Similarly, four novel lncRNAs (LNC.348, LNC.6138, LNC.11,657 and LNC.14,789) and two annotated lncRNAs (LOC106503915 and LOC108637283) were found with high degrees in the DC2 subnet. The coding genes connected to them cover all the signaling pathways in DC2, indicating that these lncRNAs are likely to be involved in the SHF degeneration. The PI3KAkt and NF-kappa B signaling are both functioning in DC2 and DC3 subnets. We found nine novel lncRNAs and four annotated lncRNAs connecting DC2 and DC3, which may play a role in the transition from telogen to early anagen.

\section{Expression differences triggered by melatonin reveal a potential signal pathway map regulating cashmere growth}

A total of 908 genes (including 70 novel lncRNAs and 48 annotated lncRNAs) were detected to have different expression patterns between the $D$ group and the $M$ group (Fig. 4 A) (Additional file 8). When treated with melatonin, 80 (24.6\%) genes in DC3 maintained the same pattern as group $\mathrm{D}$, but the expression patterns of most genes in DC1 and DC2 have changed (Fig. 4B). Among the 908 differential changing genes (DCGs), 369 genes belonging to the three clusters DC1, DC2 and DC3 were divided into three clusters MC1 (159), MC2 (144) and MC3 (66), respectively (Fig. 4C) (Additional file 9). After 


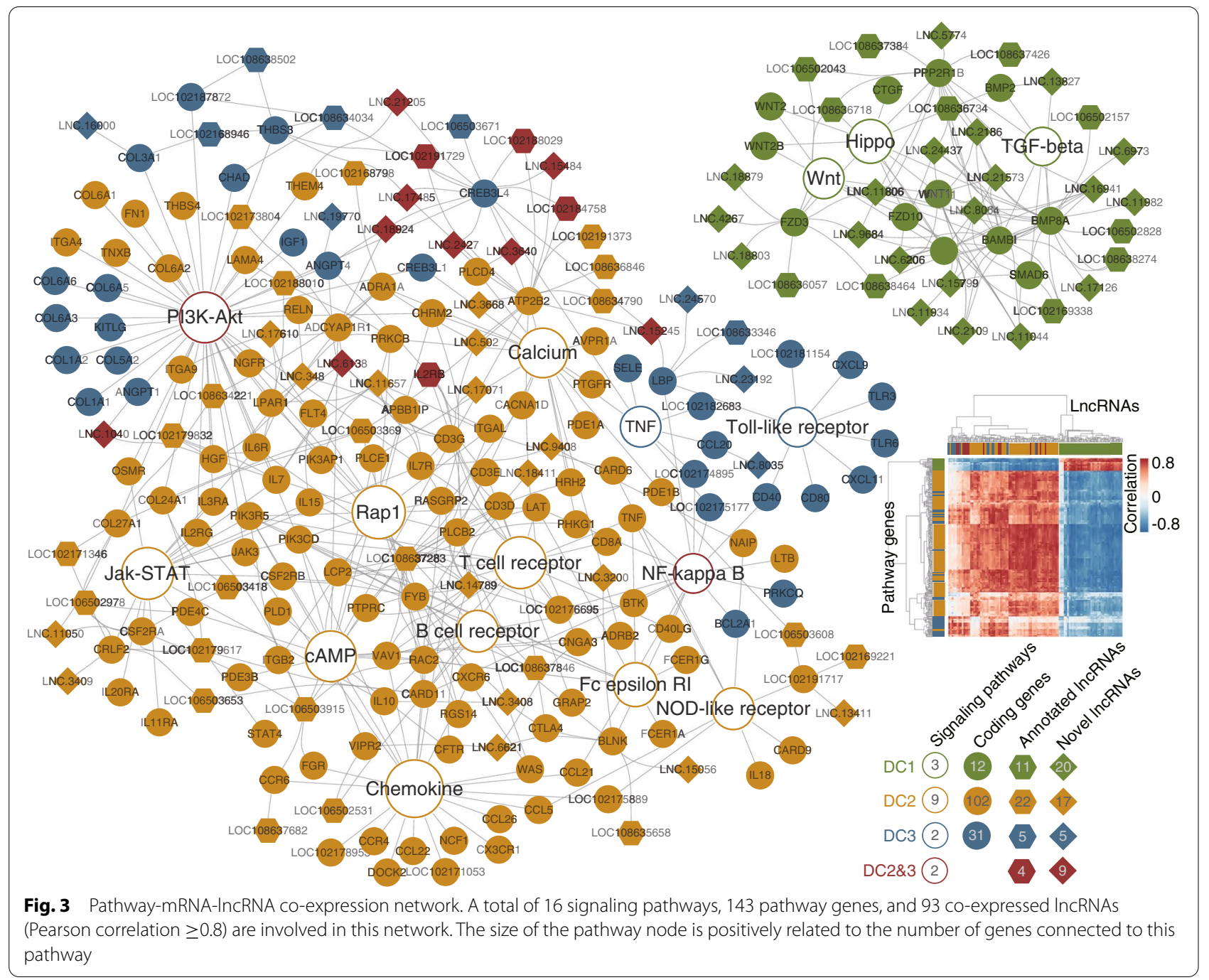

melatonin treatment, $\mathrm{MC} 1$ and $\mathrm{MC} 2$ in the $\mathrm{M}$ group lost the periodic expression fluctuations as in the $\mathrm{D}$ group (Fig. 4D). In April-May, the gene expression of MC1 dropped to the lowest while the expression of MC2 rose to the highest in the D group and shedding appeared. Pathway enrichment showed that MC1 and MC2 were involved in the promotion and regression of HFs, respectively (Fig. 4E) (Additional file 10). The six novel lncRNAs in the DC1 subnet (LNC.20,921, LNC.13,171, LNC.15,017, LNC.18,879, LNC.18,803 and LNC.20,565) disappeared in MC1, while LNC.8064 and LNC.16,941 may still play a role in the cashmere growth under melatonin treatment. In the $\mathrm{M}$ group, the expression of $\mathrm{MC} 1$ was still rising and $\mathrm{MC} 2$ was falling from January to April, which may lead to a non-resting period after shedding in the M group. Therefore, genes in MC3 (including LNC.15,245), which may be responsible for restarting HF growth, did not show a significant increase in expression in February-May. In September-November, the expression of $\mathrm{MC1}$ in the $\mathrm{M}$ group was relatively lower than that in the D group, while the expression of $\mathrm{MC} 2$ in the $M$ group was higher than that in the $\mathrm{D}$ group, which may disrupt the growth and maintenance of HFs and cause a local shedding.

To reveal the differential expression pattern every month, we used DESeq2 to obtain monthly differentially expressed genes (monthly DEGs) between melatonin and control groups with adjusted p-value (padj) $\leq 0.05$ and $\mid \log 2$ FoldChange $\mid \geq 1$. A total of 941 monthly DEGs were identified from monthly pairwise comparisons. The monthly DEGs are mainly distributed from April and September (Fig. 5 A), which exactly cover the whole anagen stage, and 906 (96\%) of them are proteincoding genes. The KEGG analysis results (Fig. 5B) (Additional file 11) showed that Hedgehog related genes ( $\mathrm{SHH}$, PTCH2, PTCH1) and Wnt related genes (FZD10, WIF1, 


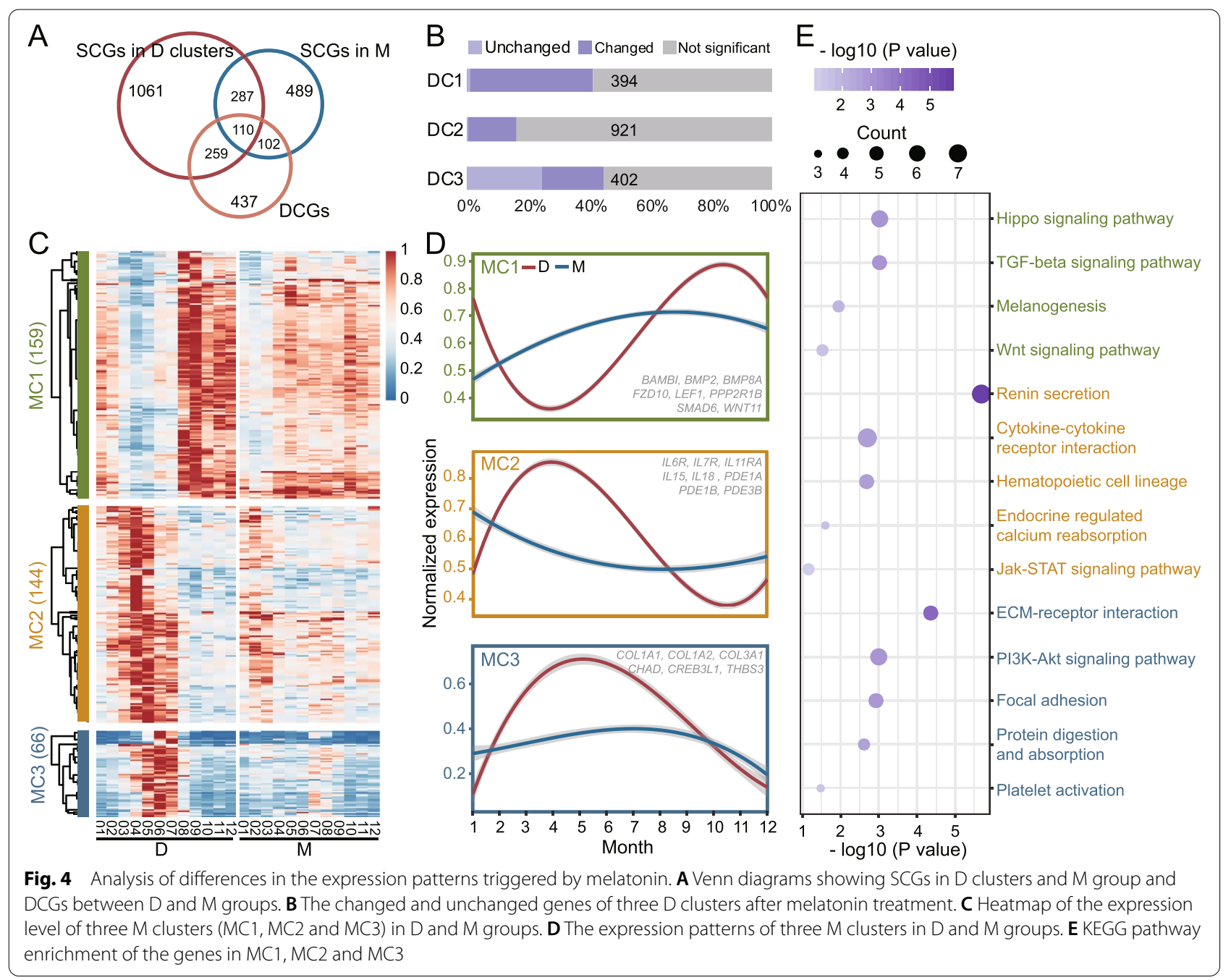

LEF1, WNT11) were up-regulated in April, while the GO results (Fig. 5 C) (Additional file 12) showed that Hedgehog genes (PTCH2, FOXE1) and other related up-regulated genes like FOXN1, HOXC13, KRT25 and KRT71 were significantly enriched from April to May. Related studies have shown that Wnt [52-54] and Shh [36, 55] signaling can promote hair follicle cell division and the initiation of anagen. Potter et al. [56] demonstrated that nude mutant gene FOXN1 is a regulatory target of HOXC13 and the knockdown of both genes could cause abnormal hair growth. Yu et al. [57] showed that a missense mutation at the helix terminus of KRT25 can cause a reduction of woolly hair. KRT71 is an inner root sheath keratin, and the mutant of KRT71 can disrupt keratin intermediate filament formation [58]. Therefore, the Hedgehog related genes (SHH, PTCH2, PTCH1, FOXE1), Wnt related genes (FZD10, WIF1, LEF1, WNT11), and other hair development related genes FOXN1, HOXC13, KRT25 and KRT71 may be responsible for the initiation of a fast anagen progressing stage from April to July. Meanwhile, the KEGG results (Fig. 5B) showed that the expression of ECM receptor interaction genes (COL6A3, THBS3, COL1A1, FRAS1, FREM1/2) was downregulated from June to July. ECM is an important matrix required for hair follicle cell growth. Xu et al. [59] showed that the amount of ECM occupied by each cell determined the volume size of dermal papilla (DP) in hair follicles, and Zhu et al. [1] demonstrated that the rapid growth of hair follicles at the anagen phase in cashmere goat required high expression of ECM and cell surface proteins. The down-regulation of ECM-related genes from June to July may contribute to the inhibition of hair follicle growth, and the down-regulation of genes involved in the Hedgehog (SHH, PTCH2) and Wnt (WNT6, NOTUM, SFRP2) pathways indicated that hair follicle growth may be inhibited in August. The expression of genes in chemokine signaling pathway (CCL17, CCL22, CCL2, LYN, RAC2, LOC102170772, PIK3CG, VAV1) was 


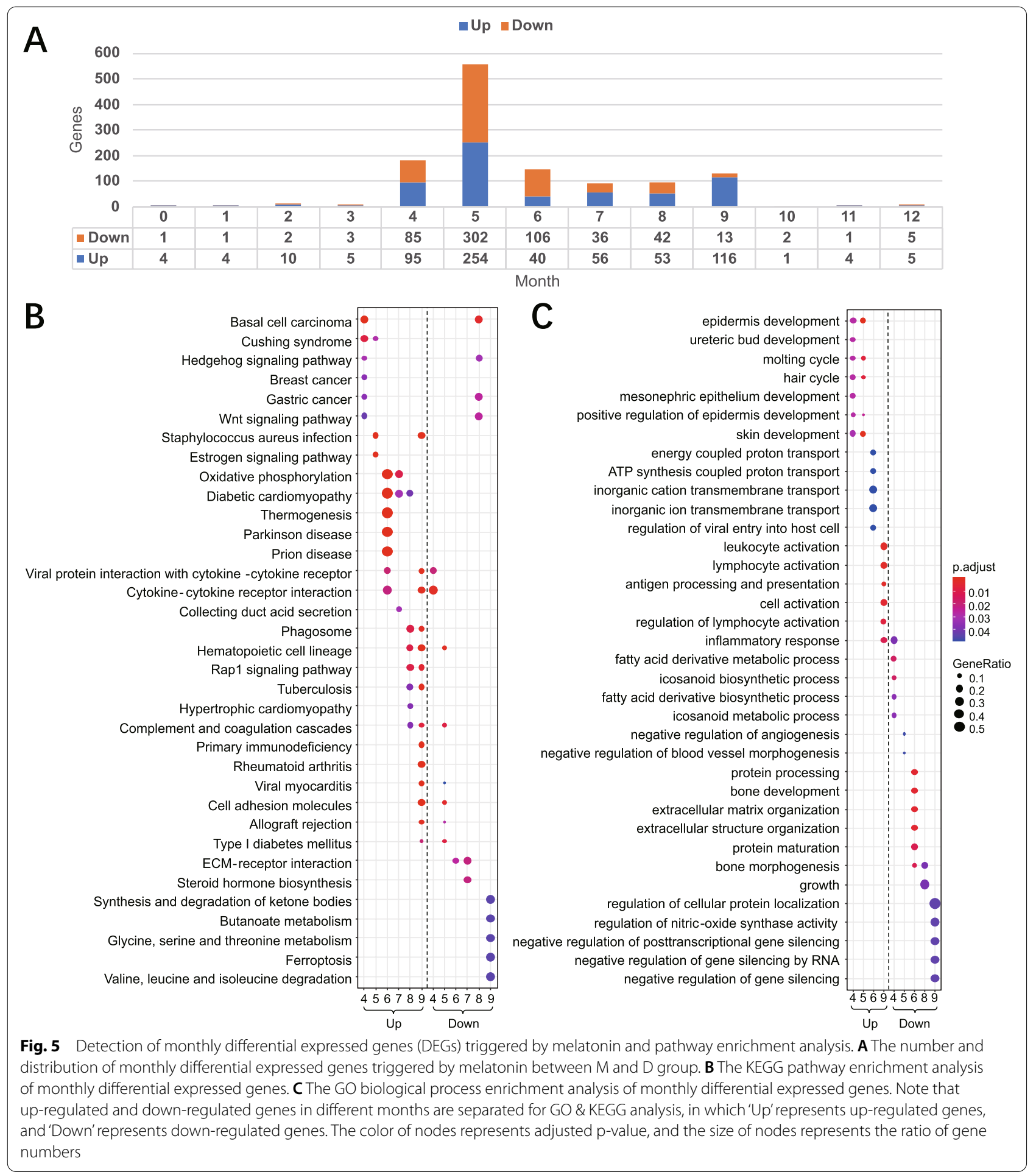

up-regulated in September. Experiments by Nagao et al. [40] have shown that chemokines can induce immune cell migration as the hair follicle enters the catagen phase, which may further promote the hair follicle apoptosis. Therefore, the downregulated ECM receptor interaction genes (COL6A3, THBS3, COL1A1, FRAS1, FREM1, FREM2), Hedgehog genes (SHH, PTCH2), Wnt genes (WNT6, NOTUM, SFRP2) and up-regulated chemokines (CCL17, CCL22, CCL2, LYN, RAC2, LOC102170772, PIK3CG, VAV1) may contribute to the second cashmere 
shedding happened from August to November. A transcriptome analysis of the melatonin-treated group with MTC knockdown experiments confirmed that melatonin can promote hair follicle development by activating the NF-kappa B pathway through promoting the expression of MTC [60]. Genes in NF-kappa B signaling pathway (CD40LG, LTB, LOC102176695, LYN, BTK) were upregulated in September, which may contribute to the hair follicle development after the second cashmere shedding. The representative GO \& KEGG enrichment results of monthly DEGs mentioned above were summarized in Table 1.

\section{Discussion}

In this study, the cashmere goat skin samples of the experimental group and the control group covered 12 months, that is, the entire hair follicle growth cycle. Many studies have used RNA-seq to explore the differences in gene expression in different growth stages of cashmere. For example, Geng et al. [61] conducted a functional analysis of the differences in gene expression between three developmental stages of hair follicles in cashmere goats, and identified key genes that are involved in the regulation of cashmere growth. Zhang et al. [13] performed transcriptome sequencing analysis on hair follicles in four seasons and explored the regulation of seasonal variation genes on the hair follicle growth cycle of the cashmere goat and milk goat. However, these studies only selected three or more stages determined by experiments or experience at the cellular level.

The gene expression pattern for 12 months can provide useful information for distinguishing different cashmere growth stages from the genetic and molecular levels. According to the cluster-month correlations in Fig. 2 C, we grouped the hair follicle growth cycle into three main stages: (1) anagen (April-October); (2) catagen and telogen (October-December and January-April); (3) telogenanagen regeneration (February-May). The corresponding gene clusters are DC1, DC2 and DC3, respectively. Some canonical pathways such as the Wnt, TGF-beta and Hippo signaling pathways are enriched in DC1. DC2 genes are mainly enriched in pathways such as Cell adhesion molecules (CAMs), Cytokine-cytokine receptor interaction, Jak-STAT, Fc epsilon RI, NOD-like receptor, Rap1, PI3K-Akt, cAMP, NF-kappa B and many immunerelated pathways. Interestingly, due to the overlap of the early anagen and telogen-anagen regeneration stages, the genes of DC3 and DC2 were partially enriched in several same pathways like CAMs, Focal adhesion, extracellular matrix (ECM)-receptor interaction, PI3K-Akt and NFkappa B signaling. Besides, by constructing a co-expression network of genes (that are enriched in key pathways) and IncRNAs in three clusters, we reveal the possible regulators for crosstalk between different signaling pathways, and unearthed novel lncRNAs that may participate in these pathways. For example, in the DC1 subnet, the Wnt and Hippo pathways are connected by six proteincoding genes (WNT2, WNT2B, WNT11, FZD3, FZD10, and LEF1), while Wnt and TGF-beta are cross-linked by the key BAMBI gene. The crosstalk between these three pathways has been proven to play an important role in cell proliferation and differentiation [33]. In addition, long non-coding RNAs (four known and nine novel) were found to bridge between DC2 and DC3 subnets, which may play a role in the transition from telogen to early anagen. One of the nine novel lncRNAs, LNC.15,245, forms a crosstalk between six pathways (PI3K-Akt, TNF, Calcium, cAMP, NF-kappa B and Toll-like receptor) by connecting three protein-coding genes (CREB3L4, ATP2B2 and LBP).

Table 1 Summary of representative GO \& KEGG enrichment of monthly DEGs

\begin{tabular}{llll}
\hline Cluster & Description & p.adjust & Gene Name \\
\hline Month4_Up & hair cycle & 0.0276 & PTCH2, FOXE1, HOXC13, FOXN1 \\
Month5_Up & hair cycle & 0.0082 & PTCH2, FOXE1, FOXN1, HOXC13, KRT71, KRT25 \\
Month4_Up & Hedgehog signaling pathway & 0.0306 & SHH, PTCH2, PTCH1 \\
Month4_Up & Wnt signaling pathway & 0.0428 & FZD10, WIF1, LEF1, WNT11 \\
Month6_Down & ECM-receptor interaction & 0.0259 & COL6A3, THBS3, FREM1, COL1A1 \\
Month7_Down & ECM-receptor interaction & 0.0218 & FREM1, FRAS1, FREM2 \\
Month8_Down & Wnt signaling pathway & 0.0257 & WNT6, NOTUM, SFRP2 \\
Month8_Down & Hedgehog signaling pathway & 0.0318 & SHH, PTCH2 \\
Month9_Up & Chemokine signaling pathway & 0.0002 & CCL17, CCL22, CCL2, LYN, RAC2, \\
Month9_Up & NF-kB signaling pathway & 0.0020 & CDC102170772, PIK3CG, VAV1
\end{tabular}

Note: The first two lines are derived from GO BP results and others are derived from KEGG results. P.adjust represents the p-values adjusted using the Benjamini \& Hochberg method 


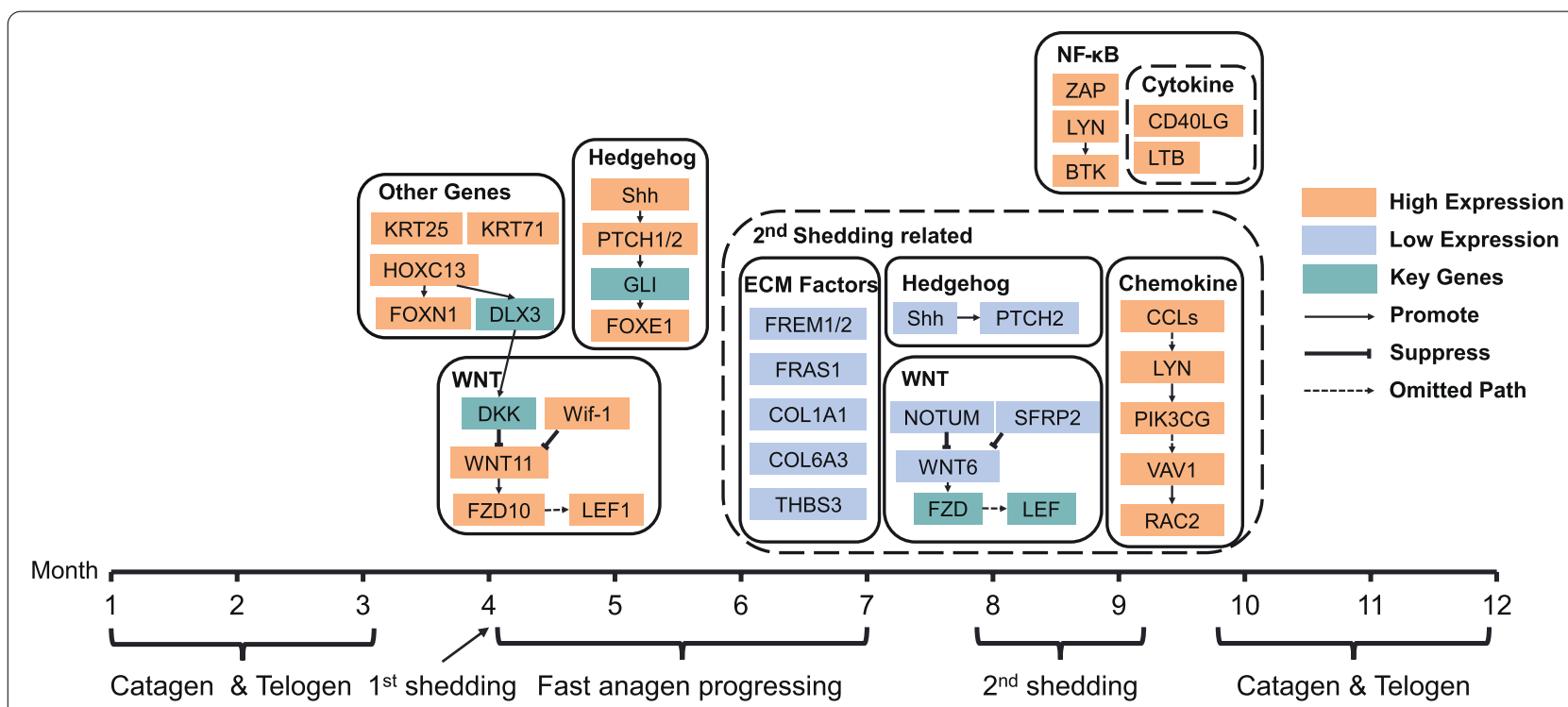

Fig. 6 The putative pathway regulation model of the hair follicle growth cycle triggered by melatonin. The signal pathways related to cashmere shedding are marked with dotted line boxes, and the other solid line boxes mark the signal pathways which may promote the hair follicle development

In addition to exploring the gene expression regulation mechanisms of the transition between different stages of the hair follicle cycle, this study also helps to unravel the role of exogenous melatonin in the specific stages of the hair follicle growth cycle. By identifying genes that exhibit different expression patterns during the hair follicle growth cycle under the stimulation of melatonin, we also obtained three gene clusters (MC1, MC2 and $\mathrm{MC} 3$ ) that may affect the hair follicle growth cycle. Among them, MC1 genes (BAMBI, BMP2, BMP8A, FZD10, LEF1, PPP2R1B, SMAD6 and WNT11) and MC2 genes (IL6R, IL7R, IL11RA, IL15, IL18, PDE1A, PDE1B and PDE3B) showed opposite periodicity in group D. However, after the melatonin treatment, this regular fluctuation has been disordered. MC3 genes (COL1A1, COL1A2, COL3A1, CHAD, CREB3L1 and THBS3) were expressed specifically in the anagen restart phase (AprMay) in group D, but there was no similarly significant expression pattern in group $M$.

The relative expression levels of monthly DEGs (Additional file 13) showed that the hair development related genes HOXC13, KRT25, KRT71, FOXN1 were generally expressed at higher levels at the beginning of fast anagen progressing period from April to May, implying that they may function to promote the initiation of anagen. Wnt genes (Wif-1, WNT11, FZD10, LEF1, NOTUM, SFRP2, WNT6) together with Hedgehog genes (SHH, PTCH1, PTCH2, FOXE1) showed higher expression levels between April and May, but decreased in August, which implied that Wnt-related genes may promote the rapid transition into anagen phase of hair follicles between April and May, and repress the growth of hair follicles on the eve of the second cashmere shedding period in August. Chemokines (CCL17, CCL22, CCL2, LYN, RAC2, LOC102170772, PIK3CG and VAV1) and NF-kB genes (ZAP70, LYN, BTK, CD40LG, LTB) were highly expressed in September. The NF- $\mathrm{B} B$ pathway may facilitate the progress of the subsequent cashmere growth phase. Meanwhile, chemokines such as LTN may promote the second cashmere shedding.

KEGG pathway could be used as a reference to demonstrate the regulatory relationships of differentially expressed genes. Taking the above results together and collating the relevant KEGG pathway visualization results (Additional file 14), here we proposed a signaling pathway diagram of melatonin influenced hair follicle growth cycle (Fig. 6), which covered the main differentially expressed genes related to cashmere growth in anagen phase from April to September. The anagen phase of melatonin-treated groups was composed of a fast anagen progressing stage and a second cashmere shedding stage. The fast anagen progressing stage was from April to July, and this phase was characterized by the occurrence of the first massive cashmere shedding at the end of April, and the rapid transition into anagen phase of hair follicles from May to July, when the quick resumption of cashmere growth appeared instead of residing in a resting period. The rapid resumption of the anagen phase of hair follicles may be due to the high expression of KRT25, HOXC13 and HOXC13's regulatory target FOXN1, high 
expression of FZD10, WIF1, LEF1, WNT11 in Wnt signaling pathway, and $\mathrm{SHH}, \mathrm{PTCH} 1, \mathrm{PTCH} 2, \mathrm{FOXE1}$ in sonic hedgehog signaling pathway. The second cashmere shedding period was from August to September. The appearance of the second cashmere shedding may not only be associated with the low expression of ECM signaling molecules such as FREM1, FREM2, FRAS1, COL1A1, COL6A3, THBS3 in June and July, sonic hedgehog signaling pathway genes such as $\mathrm{SHH}, \mathrm{PTCH} 2$ and WNT signaling pathway genes such as NOTUM, SFRP2, WNT6 in August, but also with the high expression of chemokines such as CCL, LYN, PIK3CG, VAV1, RAC2 in August. In addition, the highly expressed genes in NFkappa B signaling pathway such as CD40LG, LTB, ZAP, LYN, BTK in September may promote the subsequent growth of cashmere after the second cashmere shedding period.

\section{Conclusions}

In summary, this study systematically analyzed RNA-seq data from skin samples of cashmere goats covering the entire hair follicle growth cycle and identified a series of key regulators (including genes and lncRNAs) that may be involved in the cashmere growth processes. Based on gene expression patterns, we elucidated a more precise division of the hair follicle growth cycle from the molecular level. Some canonical signaling pathways such as Wnt, TGF- $\beta$, Jak-STAT, NF- $\mathrm{kB}$ are detected in each phase of the hair follicle growth cycle. Pairwise comparisons between the control and treated groups every month reveal a potential signaling pathway map (including Wnt, Hedgehog, ECM, Chemokines and NF- $\mathrm{B}$ signaling pathways) affecting the growing stage triggered by melatonin. Our study decodes the key regulators of the whole growth cycle of the hair follicles, laying the foundation for the control of cashmere growth and improvement of cashmere yield.

\section{Methods}

\section{Skin sample and cashmere collection}

Six female, half-sib, Inner Mongolia cashmere goats born on March 2013 with an initial weight of $33.35 \pm 1.93 \mathrm{~kg}$ were randomly divided into two groups: control group (D) and melatonin group (M). Three cashmere goats in the experimental group were implanted with melatonin (2 $\mathrm{mg} / \mathrm{kg}$ BW) every two months since December 2014. Melatonin was subcutaneously implanted at the base of the ear. Skin and cashmere samples from the six cashmere goats were collected monthly (Jan 19th, Feb 13th, Mar 21st, Apr 18th, May 20th, Jun 20th, Jul 18th, Aug 23rd, Sept 19th, Oct 20th, Nov 20th, and Dec 20th ) in a $10-\mathrm{cm}^{2}$ patch on the right mid-side of the body (behind the scapula). The subsequent skin and cashmere samples were taken as close as possible to the previous collection site, and the skin incisions were allowed to self-heal with application of the Yunnan Bai Yao (Chinese medicine: Z53020798). Immediately after collection, all skin samples were frozen in liquid nitrogen and stored at $-80{ }^{\circ} \mathrm{C}$ until use. At each sampling time point, 50 cashmeres (hairs from SHFs) were taken from each goat to measure the cashmere length.

\section{RNA extraction, library preparation and sequencing}

Total RNA from 72 collected skin samples were isolated using the TRIzol ${ }^{\mathrm{TM}}$ reagent (Invitrogen, USA) following the manufacturer's instructions (https://assets.therm ofisher.com/TFS-Assets/LSG/manuals/trizol_reagent. pdf). RNA purity was verified using the NanoPhotometer $^{\circledR}$ spectrophotometer (Implen, USA) (https://www. implen.de/wp-content/uploads/docs/Implen-NanoP hotometer-User-Manual-N120-NP80-N60-N50-C40. pdf). The ribosomal RNA (rRNA) was removed first by the Epicentre Ribo-zero ${ }^{\mathrm{TM}}$ rRNA Removal Kit (Epicentre, USA) from $3 \mu \mathrm{g}$ total RNA to prepare the RNA library. The sequencing libraries were generated using the NEBNext ${ }^{\circledR}$ Ultra ${ }^{\mathrm{TM}}$ Directional RNA Library Prep Kit for Illumina (NEB, USA) following manufacturer's instructions. Finally, the strand-specific libraries were sequenced on the Illumina Hiseq 4000 system (paired-end $150 \mathrm{bp}$ reads).

\section{Quality control}

FastQC (https://www.bioinformatics.babraham.ac.uk/ projects/fastqc/) is used to filter out low-quality residues and joints of raw reads produced by RNA-seq. The quality of clean reads (Q20, Q30, and GC content) were detected. The high quality clean reads were retained for subsequent analysis.

\section{Read alignment and transcriptome assembly}

Clean reads were aligned to the goat genome (NCBI assembly ARS1) using HISAT2 [62] under default settings. The mapped reads of each sample were assembled by StringTie [62] in a reference-based mode. All transcripts assembled were merged into a Gene transfer format (GTF) file.

\section{Novel IncRNA identification}

The transcripts without matching the known annotations were selected to identify novel lncRNAs through the following steps: (1) transcripts less than $200 \mathrm{bp}$ in length were removed; (2) transcripts with exon number less than 2 were removed; (3) the coding potential of each transcript was predicted using CPC2 [63] and CNCI [64], and transcripts predicted as "noncoding" were imported into Pfam Scan (http://ftp.ebi.ac.uk/pub/databases/Pfam/ 
Tools/) to filter out those with known protein family domains (Pfam release 30).

\section{Reads counting and time-series differential expression analysis}

All coding genes and lncRNA genes were included to calculate the count matrix using htseq-count [65]. The likelihood ratio test (LRT) in DESeq2 [66] was used to identify the seasonal changing genes (SCGs) in both control and melatonin group with model " time" versus " $\sim 1$ ". The SCGs were defined as genes with significant expression changes throughout the hair follicle growth cycle. Initially, the count matrix was normalized by the size factors estimated by DESeq2 and the median of the normalized gene expression values at each time point were used to calculate the fold change $f_{i}$ :

$$
\begin{aligned}
\mu_{i t} & =\operatorname{median}_{j}\left(G_{i j t}\right) \\
f_{i} & =\frac{\max _{t}\left(\mu_{i t}\right)}{\min _{t}\left(\mu_{i t}\right)+1}
\end{aligned}
$$

Where $G_{i j t}$ is the normalized expression value of gene $i$ in goatjat sampling timet. The thresholds for adjusted p-value and fold change were set to 0.05 and 2, respectively. Besides, the differential changing genes (DCGs) between control and melatonin group were detected using LRT with model " time + condition + time: condition" versus " $\sim$ time + condition".

\section{Gene cluster detection}

The SCGs detected in control group were included for weighted gene co-expression network analysis (WGCNA) [67]. Pearson correlations between gene clusters and trait data (months) were calculated. The minimum cluster size was set to 50 , and clusters with a tree height less than 0.25 in the dendrogram were merged.

\section{Pathway enrichment}

The DAVID database [68] was used to identify the significantly enriched Kyoto Encyclopedia of Genes and Genomes (KEGG) pathways [69] for gene modules detected by WGCNA. The p-value threshold for enrichment was set to 0.05 . The pathways enriched in infectious diseases and cancer were removed.

Monthly DEGs were annotated with Gene Ontology (GO) [70] biological process (BP) and KEGG analysis. The annotations were all achieved with clusterProfiler [71] package, with q-value $\leq 0.05$ for $\mathrm{GO}$ and $\mathrm{p}$-value $\leq$ 0.05 for KEGG. Annotation information was retrieved from the Ensembl database using AnnotationHub (https://bioconductor.org/packages/release/bioc/html/
AnnotationHub.html) to generate an OrgDb annotation file.

\section{Pathway-mRNA-IncRNA network construction}

We selected genes enriched in key signaling pathways as sources, and co-expressed lncRNA genes as targets to construct the pathway-mRNA-lncRNA network. The Pearson correlations between pathway genes and lncRNA genes were calculated, and those mRNA-lncRNA pairs with correlation higher than 0.8 were selected to construct the network. CytoScape [72] was used to visualize the network.

\section{Calculation of relative gene expression for monthly DEGs}

To observe the effects of melatonin on the expression of representative differentially expressed gene, we plotted relative expression boxplots to visually compare the expression trends of these genes. The horizontal axis of the boxplot represents months, and the vertical axis represents relative expression levels, which were calculated as follows:

$$
\text { relativeexpressionlevel }=\ln \left(\frac{\text { GeneCountsinMelatonin }}{\text { GeneCountsinControl }}\right)
$$

The gene counts were normalized with total counts in different groups. Three relative expression values per month were used for boxplot visualization.

\section{Abbreviations}

SCG: Seasonal changing gene; CAM: Cell adhesion molecule; DEG: Differentially expressed gene; Monthly DEG: Monthly differentially expressed gene; ECM: Extracellular matrix; HF: Hair follicle; SHF: Secondary hair follicle; BMP: Bone morphogenetic protein; SHH: Sonic hedgehog; FGF: Fibroblast growth factor; TGF: Transforming growth factor; KRT: Keratin; KRTAP: Keratin-associated protein; IncRNA: Long noncoding RNA; miRNA: MicroRNA; WGCNA: Weighted gene co-expression network analysis; KEGG: Kyoto Encyclopedia of Genes and Genomes; GO: Gene Ontology; BP: Biological process; PCA: Principal components analysis; LEF: Lymphoid enhancer binding factor; IL: Interleukin; DKK: Dickkopf

\section{Supplementary Information}

The online version contains supplementary material available at https://doi. org/10.1186/s12864-022-08331-z.

Additional file 1. Read counts of all detected genes in 72 samples.

Additional file 2. Predicted IncRNAs.

Additional file 3. Differentially expressed genes in the control group. Additional file 4. Differentially expressed genes in the melatonin-treated group.

Additional file 5. Detailed genes of 3 clusters in the control group (DC1, DC2 and DC3).

Additional file 6. The KEGG enrichment results of genes in DC1, DC2 and DC3.

Additional file 7. The Pearson correlations between genes and IncRNAs. 
Additional file 8. Genes with different expression patterns between the control and melatonin-treated groups.

Additional file 9. Detailed genes of 3 clusters in the melatonin-treated group (MC1, MC2 and MC3).

Additional file 10. The KEGG enrichment results of genes in MC1, MC2 and $\mathrm{MC}$.

Additional file 11. The KEGG analysis results of monthly DEGs.

Additional file 12. The GO analysis results of monthly DEGs.

Additional file 13. Boxplots of relative expression levels of monthly DEGs.

Additional file 14. KEGG Pathway Maps obtained from KEGG (https:// www.kegg.jp/kegg/pathway.html).

\section{Acknowledgements}

The authors are thankful for the samples provided by the Hanshan White Cashmere Goat Breeding Farm.

\section{Authors' contributions}

C. L. conducted the research, C. F. and G. M. analysed the data and prepare the manuscript, S. F. helped RNA-seq and data processing, J. L. and W. Z. provided the test platform, M. C. supervised data analysis and manuscript revising. The authors read and approved the final manuscript.

\section{Funding}

This work has been funded under the National Natural Science Foundation of China (31402052), and the Natural Science Foundation of Inner Mongolia (2019MS03078, 2019BS03006, 2019LH03012), and the Special Open Project on the Construction of Mongolian Pharmacy Doctoral Station for National Service with Special Needs (MDMYBJ2019004), and the Doctoral Scientific Research Foundation of Inner Mongolia University for Nationalities (BS308) provided support for the design of the study and data collection.

\section{Availability of data and materials}

All data generated or analyzed during this study are included in this article and Supplementary files, as well as in Genome Sequence Archive (https:// ngdc.cncb.ac.cn/gsa/) under accession number CRA005496.

\section{Declarations}

\section{Ethics approval and consent to participate}

The skin samples in this study were collected from the Inner Mongolia Cashmere Goats. All experimental procedures were approved by the standards of the Animal Care and Use Committee in Inner Mongolia University for Nationalities, China. All experimental methods were in accordance to the "Guidelines for Experimental Animals" of the Ministry of Science and Technology (Beijing, China). All methods are reported in accordance with ARRIVE guidelines (https://arriveguidelines.org).

\section{Consent for publication}

Not applicable.

\section{Competing interests}

The authors declare that they have no competing interests.

\section{Author details}

'College of Animal Science and Technology, Inner Mongolia Minzu University, Tongliao 028000, China. ${ }^{2}$ College of Life Sciences, Zhejiang University, Hangzhou 310058, China. ${ }^{3}$ Inner Mongolia Academy of Agricultural \& Animal Husbandry Sciences, Hohhot 010018, China. ${ }^{4}$ College of Life Science and Food Engineering, Inner Mongolia Minzu University, Tongliao 028000, China. ${ }^{5}$ College of Animal Science, Inner Mongolia Agricultural University, Hohhot 010018, China.

Received: 25 September 2021 Accepted: 19 January 2022 Published online: 16 February 2022

\section{References}

1. Zhu B, Xu T, Yuan J, Guo X, Liu D. Transcriptome sequencing reveals differences between primary and secondary hair follicle-derived dermal papilla cells of the cashmere goat (Capra hircus). PLoS ONE. 2013;8(9):e76282

2. Bykov YS, Schaffer M, Dodonova SO, Albert S, Plitzko JM, Baumeister W, Engel BD, Briggs JA: The structure of the COPI coat determined within the cell. eLife 2017,6

3. Pin D, Cachon T, Carozzo C. Determination of the depth of excision using a dermatome (Aesculap ${ }^{\circledR}$ ) to export all hair follicle bulbs from a donor site in the dog. J VET MED A. 2007;54(9):539-41.

4. McCarthy B. Specialty animal fibers. Textiles. 1998;20(1):6-8.

5. Yang F, Liu Z, Zhao M, Mu Q, Che T, Xie Y, Ma L, Mi L, Li J, Zhao Y: Skin transcriptome reveals the periodic changes in genes underlying cashmere (ground hair) follicle transition in cashmere goats. BMC Genomics 2020, 21(1).

6. Klören WRL, Norton BW. Melatonin and fleece growth in Australian cashmere goats. Small Rumin Res. 1995;17(2):179-85.

7. Betteridge K, Welch R, Pomroy W, Lapwood K, Devantier B: Out of season cashmere growth in feral goats. In: Proceedings of the second international cashmere conference: 1987; Christchurch, New Zealand: Lincoln College Press; 1987: 137-143.

8. Litherland AJ, Paterson DJ, Parry AL, Dick HB, Staples LD. Melatonin for cashmere production. Proc New Zealand Soc Anim Prod. 1990;50:339-43.

9. Welch RAS, Gurnsey MP, Betteridge K, Mitchell RJ: Goat fibre response to melatonin given in spring in two consecutive years. Proc New Zealand Soc Anim Prod 1990, 50:335-338.

10. Yang $\mathrm{CH}$, Duan CH, Wu ZY, Li Y, Luan YY, Fu XJ, Zhang CX, Zhang W. Effects of melatonin administration to cashmere goats on cashmere production and hair follicle characteristics in two consecutive cashmere growth cycles. Domest Anim Endocrinol. 2021;74:106534.

11. Duan $\mathrm{CH}, \mathrm{Xu}$ JH, Sun CM, Jia ZH, Zhang W: Effects of melatonin implantation on cashmere yield, fibre characteristics, duration of cashmere growth as well as growth and reproductive performance of Inner Mongolian cashmere goats. J Anim Sci Biotechnol 2015, 6.

12. Yurong L, Wenbin F, Changqing L. Histomorphology research of the secondary follicle cycling of Inner Mongolia Cashmere goat. Scientia Agricultura Sinica. 2008;41(11):3920-6.

13. Su R, Fan YX, Qiao X, Li XK, Zhang L, Li C, Li JQ: Transcriptomic analysis reveals critical genes for the hair follicle of Inner Mongolia Cashmere goat from catagen to telogen. PLoS One 2018, 13(10).

14. Tsai S-Y, Sennett R, Rezza A, Clavel C, Grisanti L, Zemla R, Najam S, Rendl M. Wnt/ $\beta$-catenin signaling in dermal condensates is required for hair follicle formation. Dev Biol. 2014;385(2):179-88.

15. Enshell-Seijffers D, Lindon C, Kashiwagi M, Morgan BA. $\beta$-catenin activity in the dermal papilla regulates morphogenesis and regeneration of hair. Dev Cell. 2010;18(4):633-42.

16. Veltri A, Lang C, Lien W-H. Concise review: Wnt signaling pathways in skin development and epidermal stem cells. Stem Cells. 2018;36(1):22-35.

17. Yuhki M: BMPR1A signaling is necessary for hair follicle cycling and hair shaft differentiation in mice. Development 2004, 131(8):1825-1833.

18. Kulessa $\mathrm{H}$ : Inhibition of BMP signaling affects growth and differentiation in the anagen hair follicle. The EMBO Journal 2000, 19(24):6664-6674.

19. Woo WM, Zhen HH, Oro AE: Shh maintains dermal papilla identity and hair morphogenesis via a Noggin-Shh regulatory loop. Genes Dev 2012, 26(11):1235-1246.

20. Żak M, Klis SFL, Grolman W: The Wnt and Notch signalling pathways in the developing cochlea: formation of hair cells and induction of regenerative potential. Int J Dev Neurosci 2015, 47(Part_B):247-258.

21. Rosenquist TA, Martin GR: Fibroblast growth factor signalling in the hair growth cycle: expression of the fibroblast growth factor receptor and ligand genes in the murine hair follicle. Dev Dyn 1996, 205(4):379-386.

22. Choi YM, Choi SY, Kim H, Kim J, Ki MS, An I-S, Jung J: TGF $\beta$ family mimetic peptide promotes proliferation of human hair follicle dermal papilla cells and hair growth in C57BL/6 mice. Biomed Dermatol 2018, 2(1).

23. Wang J, Sui J, Mao C, Li X, Chen X, Liang C, Wang X, Wang S-H, Jia C: Identification of key pathways and genes related to the development of hair follicle cycle in Cashmere goats. Genes 2021, 12(2):180. 
24. Kung JT, Colognori D, Lee JT: Long noncoding RNAs: past, present, and future. Genetics 2013, 193(3):651-669.

25. Nie Y, Li S, Zheng X, Chen W, Li X, Liu Z, Hu Y, Qiao H, Qi Q, Pei Q et al: Transcriptome reveals long non-coding RNAs and mRNAs involved in primary wool follicle induction in carpet sheep fetal skin. Front Physiol 2018, 9

26. Yin R, Wang Y, Zhao S, Yin R, Bai M, Wang Z, Zhu Y, Cong Y, Liu H, Bai W: LncRNA-599554 sponges miR-15a-5p to contribute inductive ability of dermal papilla cells through positively regulating the expression of Wnt3a in cashmere goat. Electron J Biotechnol 2020, 45.

27. Wang S, Ge W, Luo Z, Guo Y, Jiao B, Qu L, Zhang Z, Wang X: Integrated analysis of coding genes and non-coding RNAs during hair follicle cycle of cashmere goat (Capra hircus). BMC Genomics 2017, 18(1).

28. Wang J, Che L, Hickford J, Zhou H, Hao Z, Luo Y, Hu J, Liu X, Li S: Identification of the caprine keratin-associated protein 20-2 (KAP20-2) gene and its effect on cashmere traits. Genes 2017, 8(11):328.

29. Sulayman A, Tian K, Huang X, Tian Y, Xu X, Fu X, Zhao B, Wu W, Wang $D$, Yasin A et al: Genome-wide identification and characterization of long non-coding RNAs expressed during sheep fetal and postnatal hair follicle development. Sci Rep 2019, 9(1).

30. Oshimori N, Fuchs E: Paracrine TGF-beta signaling counterbalances BMP-mediated repression in hair follicle stem cell activation. Cell Stem Cell 2012, 10(1):63-75.

31. Rishikaysh P, Dev K, Diaz D, Qureshi WM, Filip S, Mokry J: Signaling involved in hair follicle morphogenesis and development. Int J Mol Sci 2014, 15(1):1647-1670.

32. Zhang $Y$, Yu J, Shi $C$, Huang $Y$, Wang $Y$, Yang $T$, Yang J: Lef1 contributes to the differentiation of bulge stem cells by nuclear translocation and cross-talk with the Notch signaling pathway. Int J Medical Sci 2013, 10(6):738-746.

33. Attisano L, Wrana JL: Signal integration in TGF-beta, WNT, and Hippo pathways. F1000Prime Rep 2013, 5:17.

34. Hwang J, Mehrani T, Millar SE, Morasso MI: Dl×3 is a crucial regulator of hair follicle differentiation and cycling. Development 2008, 135(18):3149-3159.

35. Guo T, Han J, Yuan C, Liu J, Niu C, Lu Z, Yue Y, Yang B: Comparative proteomics reveals genetic mechanisms underlying secondary hair follicle development in fine wool sheep during the fetal stage. J Proteomics 2020, 223:103827.

36. St-Jacques B, Dassule H, Karavanova I, Botchkarev V, Li J, Danielian P, McMahon J, Lewis P, Paus R, McMahon A: Sonic hedgehog signaling is essential for hair development. Curr Biol 1998, 8(19):1058-1069.

37. Nanba D, Nakanishi Y, Hieda Y: Role of Sonic hedgehog signaling in epithelial and mesenchymal development of hair follicles in an organ culture of embryonic mouse skin. Dev Growth Differ 2003, 45(3):231-239.

38. Abe Y, Tanaka N: Roles of the hedgehog signaling pathway in epidermal and hair follicle development, homeostasis, and cancer. J Dev Biol 2017, 5(4).

39. Mueller SN, Germain RN: Stromal cell contributions to the homeostasis and functionality of the immune system. Nat Rev Immunol 2009, 9(9):618-629.

40. Nagao K, Kobayashi T, Moro K, Ohyama M, Adachi T, Kitashima DY, Ueha S, Horiuchi K, Tanizaki H, Kabashima K et al: Stress-induced production of chemokines by hair follicles regulates the trafficking of dendritic cells in skin. Nat Immunol 2012, 13(8):744-752.

41. Scharschmidt TC, Vasquez KS, Pauli ML, Leitner EG, Chu K, Truong HA, Lowe MM, Sanchez Rodriguez R, Ali N, Laszik ZG et al: Commensal microbes and hair follicle morphogenesis coordinately drive treg migration into neonatal skin. Cell Host Microbe 2017, 21(4):467-477 e465.

42. Ariel A, Fredman G, Sun YP, Kantarci A, Van Dyke TE, Luster AD, Serhan $\mathrm{CN}$ : Apoptotic neutrophils and T cells sequester chemokines during immune response resolution through modulation of CCR5 expression. Nat Immunol 2006, 7(11):1209-1216.

43. Harel S, Higgins CA, Cerise JE, Dai Z, Chen JC, Clynes R, Christiano AM: Pharmacologic inhibition of JAK-STAT signaling promotes hair growth. Sci Adv 2015, 1(9):e1500973.

44. Wang ECE, Dai Z, Ferrante AW, Drake CG, Christiano AM: A subset of TREM2 $\left(^{+}\right)$dermal macrophages secretes oncostatin $M$ to maintain hair follicle stem cell quiescence and inhibit hair growth. Cell Stem Cell 2019, 24(4):654-669 e656.
45. Kim JE, Lee YJ, Park HR, Lee DG, Jeong KH, Kang H: The effect of JAK inhibitor on the survival, anagen re-entry, and hair follicle immune privilege restoration in human dermal papilla cells. Int J Mol Sci 2020, 21(14).

46. Kwack MH, Kim MK, Kim JC, Sung YK: Dickkopf 1 promotes regression of hair follicles. J Invest Dermatol 2012, 132(6):1554-1560.

47. Kwack MH, Lee JH, Seo CH, Kim JC, Kim MK, Sung YK: Dickkopf-1 is involved in dexamethasone-mediated hair follicle regression. Exp Dermatol 2017, 26(10):952-954.

48. Grosbois J, Demeestere I: Dynamics of PI3K and Hippo signaling pathways during in vitro human follicle activation. Hum Reprod 2018, 33(9): 1705-1714.

49. Chen Y, Fan Z, Wang X, Mo M, Zeng SB, Xu RH, Wang X, Wu Y: PI3K/Akt signaling pathway is essential for de novo hair follicle regeneration. Stem Cell Res Ther 2020, 11(1):144.

50. Nelson AM, Reddy SK, Ratliff TS, Hossain MZ, Katseff AS, Zhu AS, Chang E, Resnik SR, Page C, Kim D et al: dsRNA Released by Tissue Damage Activates TLR3 to Drive Skin Regeneration. Cell Stem Cell 2015, 17(2):139-151.

51. Wang X, Wang J, Guo L, Wang X, Chen H, Wang X, Liu J, Tredget EE, Wu $Y$ : Self-assembling peptide hydrogel scaffolds support stem cell-based hair follicle regeneration. Nanomedicine 2016, 12(7):2115-2125.

52. Millar SE, Willert K, Salinas PC, Roelink H, Nusse R, Sussman DJ, Barsh GS: WNT signaling in the control of hair growth and structure. Dev Biol 1999, 207(1):133-149.

53. Stenn KS, Paus R: Controls of hair follicle cycling. Physiol Rev 2001 , 81(1):449-494.

54. Li Y-H, Zhang K, Yang K, Ye J-X, Xing Y-Z, Guo H-Y, Deng F, Lian X-H, Yang T: Adenovirus-mediated Wnt10b overexpression induces hair follicle regeneration. J Invest Dermatol 2013, 133(1):42-48.

55. Mill P: Sonic hedgehog-dependent activation of Gli2 is essential for embryonic hair follicle development. Genes Dev 2003, 17(2):282-294.

56. Potter CS, Pruett ND, Kern MJ, Baybo MA, Godwin AR, Potter KA, Peterson RL, Sundberg JP, Awgulewitsch A: The nude mutant gene FOXN1 is a HOXC13 regulatory target during hair follicle and nail differentiation. I Invest Dermatol 2011, 131(4):828-837.

57. Yu X, Chen F, Ni C, Zhang G, Zheng L, Zhang J, Li C, Sandilands A, Yao Z, Li M: A missense mutation within the helix termination motif of KRT25 causes autosomal dominant woolly hair/hypotrichosis. J Invest Dermatol 2018, 138(1):230-233.

58. Fujimoto A, Farooq M, Fujikawa H, Inoue A, Ohyama M, Ehama R, Nakanishi J, Hagihara M, Iwabuchi T, Aoki J et al: A missense mutation within the helix initiation motif of the keratin K71 gene underlies autosomal dominant woolly hair/hypotrichosis. J Invest Dermatol 2012, 132(10):2342-2349

59. Xu T, Guo X, Wang H, Hao F, Du X, Gao X, Liu D: Differential gene expression analysis between anagen and telogen of Capra hircus skin based on the de novo assembled transcriptome sequence. Gene 2013, 520(1):30-38

60. Jin M, Cao M, Cao Q, Piao J, Zhao F, Piao Ja: Long noncoding RNA and gene expression analysis of melatonin-exposed Liaoning cashmere goat fibroblasts indicating cashmere growth. Naturwissenschaften 2018, 105(9):60.

61. Geng RQ, Yuan C, Chen YL: Exploring differentially expressed genes by RNA-seq in cashmere goat (Capra hircus) skin during hair follicle development and cycling. PLoS One 2013, 8(4).

62. Pertea M, Kim D, Pertea GM, Leek JT, Salzberg SL: Transcript-level expression analysis of RNA-seq experiments with HISAT, StringTie and Ballgown. Nat Protoc 2016, $11(9): 1650$.

63. Kang Y-J, Yang D-C, Kong L, Hou M, Meng Y-Q, Wei L, Gao G: CPC2: a fast and accurate coding potential calculator based on sequence intrinsic features. Nucleic Acids Res 2017, 45(W1):W12-W16.

64. Sun L, Luo H, Bu D, Zhao G, Yu K, Zhang C, Liu Y, Chen R, Zhao Y: Utilizing sequence intrinsic composition to classify protein-coding and long non-coding transcripts. Nucleic Acids Res 2013, 41(17):e166.

65. Anders S, Pyl PT, Huber W: HTSeq-a Python framework to work with high-throughput sequencing data. Bioinformatics 2015, 31(2):166-169.

66. Love MI, Huber W, Anders S: Moderated estimation of fold change and dispersion for RNA-seq data with DESeq2. Genome Biol 2014, 15(12):1-21. 
67. Langfelder P, Horvath S: WGCNA: an R package for weighted correlation network analysis. BMC Bioinformatics 2008, 9(1):1-13.

68. Huang DW, Sherman BT, Tan Q, Kir J, Liu D, Bryant D, Guo Y, Stephens R, Baseler MW, Lane HC: DAVID Bioinformatics Resources: expanded annotation database and novel algorithms to better extract biology from large gene lists. Nucleic Acids Res 2007, 35:W169-W175.

69. Kanehisa M, Goto S: KEGG: kyoto encyclopedia of genes and genomes. Nucleic Acids Res 2000, 28(1):27-30.

70. Ashburner M, Ball CA, Blake JA, Botstein D, Butler H, Cherry JM, Davis AP, Dolinski K, Dwight SS, Eppig JT et al: Gene Ontology: Tool for the unification of biology. Nat Genet 2000, 25(1):25-29.

71. Yu G, Wang L-G, Han Y, He Q-Y: ClusterProfiler: an R package for comparing biological themes among gene clusters. OMICS: I Integrative Biol 2012, 16(5):284-287.

72. Shannon P, Markiel A, Ozier O, Baliga NS, Wang JT, Ramage D, Amin N, Schwikowski B, Ideker T: Cytoscape: a software environment for integrated models of biomolecular interaction networks. Genome Res 2003 , 13(11):2498-2504.

\section{Publisher's Note}

Springer Nature remains neutral with regard to jurisdictional claims in published maps and institutional affiliations.

- fast, convenient online submission

- thorough peer review by experienced researchers in your field

- rapid publication on acceptance

- support for research data, including large and complex data types

- gold Open Access which fosters wider collaboration and increased citations

- maximum visibility for your research: over $100 \mathrm{M}$ website views per year

At BMC, research is always in progress.

Learn more biomedcentral.com/submissions 